\title{
Clinical neuroimaging markers of response to treatment in mood disorders
}

Michele Porcu ${ }^{1}$ - Antonella Balestrieri ${ }^{1}$ - Paolo Siotto ${ }^{2}$ - Pierleone Lucatelli $^{3}$ Michele Anzidei ${ }^{4}$ - Jasjit S Suri ${ }^{5}$ - Fulvio Zaccagna ${ }^{4}-$ Giovanni Maria Argiolas $^{2}$ - Luca $\mathrm{Saba}^{1 *}$

${ }^{1}$ Department of Radiology - AOU of Cagliari - SS 554 Monserrato (CA) - ITALY

${ }^{2}$ Department of Radiology - AOB Azienda Ospedaliera Brotzu - (CA) - ITALY

${ }^{3}$ Vascular and Interventional Radiology Unit, Department of Radiological, Oncological and Anatomo-Pathological Sciences, Sapienza University of Rome, Viale Regina Elena 324, 00161, Rome (ITALY)

${ }^{4}$ Department of Radiological, Oncological and Anatomo-Pathological Sciences, Sapienza University of Rome, Viale Regina Elena 324, 00161, Rome (ITALY)

${ }^{5}$ Diagnostic and Monitoring Division, AtheroPoint ${ }^{\mathrm{TM}}$, Roseville, CA, USA; Electrical Engineering Department, Idaho State University (Aff.), Pocatello, ID, USA

Corresponding author Prof Luca Saba: lucasaba@tiscali.it 


\section{A. Introduction}

Mood disorders (MD) are important and frequent psychiatric pathologies, and the management of the patients affected by these conditions represent an important factor of disability and a huge problem in social terms and an economic burden.

Several epidemiological studies [1-5] have illustrated the high incidence and prevalence of these disorders all around the world. European Study of the Epidemiology of Mental Disorders (ESEMeD) recently conducted a study involving several European nations and concluded that patients who had 12 month history of mental or emotional discomforts had a 36\% chance of being MD [1].

Different from other pathological conditions, such as infective or surgical diseases, due to intrinsic complexity of the human brain, and of its internal connections makes the study of the psychiatric disorders really difficult with the actual scientific instruments. Further, we cannot forget that the external environment plays a pivotal role in the development and progression of these diseases.

During the last decades many "post mortem" studies allowed to understand the gross anatomic-pathological alterations at the base of these pathologies. This included the differences in dimensions of the brain structures, number and morphology of neuronal cells, distribution of whitegray matter, and in the molecular and genetic expressions of specific molecules inside and on the surface of the cells. The highest limitation of these investigations are the fact that they are, for definition, post-mortem studies, which can give us some information about the end-stage of the illness, but with very low data about the first pathophysiological events [6].

The "in-vivo" studies can help researchers to understand the first events at the base of the development of the pathology and to identify the molecular and non-molecular targets of therapies, but they have strong limitations due to the fact that human brain circuits themselves are difficult to be reproduced in animal models. Besides these challenges, they are difficult to be selectively studied with the modern imaging (such as Magnetic Resonance and Positron Emitted Tomography / Computed Tomography) and non-imaging (such as electroencephalography, magnetoencephalography, transcranial magnetic stimulation and evoked potentials) methods [6].

All these factors make difficult to fully understand the aetiology and pathophysiology of these disorders, and consequently make difficult not only in the development, but also the monitoring of the actions of therapies, which according to clinical observations have been demonstrated effective in the treatment.

In this review, we will focus our attention on the actual state-of-the-art of role of imaging in monitoring of treatment of MD, underlying that up to date there are still not standardized imaging markers available in clinical practice; all the studies mentioned in this review are clinical studies performed on humans. We will analyse briefly the actual classification of MD; then we will focus on 
the "in vivo" imaging modalities used in research and clinical activity, the current knowledge about the neural models at the base of MD. The last part of the review focuses on analysis of the principle markers of response to the treatment according to the type of treatment used and to the imaging techniques adopted.

\section{B. MD: definition and classifications.}

Historically, the definition and the diagnosis of psychiatric disorders have been quite difficult to assess. In the last decades, a great work has been made by the international psychiatric associations in order to standardize stable criteria for the diagnosis of MD; these criteria are based almost exclusively on clinical observations, and the pathophysiological mechanisms at the base of these conditions are still debate because of the difficulties exposed in the earlier part of this manuscript.

Nowadays, the diagnosis of psychiatric disorders is mostly made according to the data of the Diagnostic and Statistical Manual (DSM) of Mental Disorder, released at its $5^{\text {th }}$ edition in 2013 (DSM$5^{\text {th) }}$ [7] and largely integrated with the criteria of the International Classification of Disease released in its $10^{\text {th }}$ edition (ICD-10) in 1990 by the World Health Organization (WHO).

According to the previous edition of DSM, the $4^{\text {th }}$, released in 1994 (DSM-4th) [8], the MD were grouped into a specific category of disorders in which the disturbance of the mood is the main future, and this was characterized by the appearance of different mood episodes (i.e. Major Depressive, Manic, Mixed or Hypomanic episode) at different times [8]. Both the episodes and the disorders have to satisfy specific diagnostic criteria, based on the features of the symptoms, on the exclusion of effects induced by concomitant drugs, and on the exclusion of other general medical conditions responsible of the symptoms [8]. Among the principle MD, the two main and more frequent are the Bipolar Disorder (BD), in particular type I (BD-I, the most studied) and type II (BD-II), and the Major Depressive Disorder (MDD) also known as Unipolar Disorder (UD).

The above mentioned classification was changed in the DSM-5 th $[8,9]$ : now bipolar and related disorders are grouped separately from the other depressive disorders, and they are considered pathologies with intermediate features between schizophrenia and psychotic related disorders on one side (i.e. psychoticism and disinhibition) and the "internalizing" disorders such as depressive disorders (i.e., negative affectivity) on the other side [8-10].

Despite this new updated classification, for convenience in the next paragraphs we will continue our analysis talking about "MD" as referred in the DSM-4th classification. This is because the greatest part of the studies published up to date has been published before the introduction of the new DSM-5th. 


\section{C. "In vivo" imaging studies in MD: a brief exposure of the main features and limits.}

Differences in anatomical structures, molecular compositions and functional activity among different regions of the Central Nervous System (CNS), or of the same CNS region between healthy and illness patients or before and after a treatment, can give useful information in order to understand not only the aetiology and pathophysiology of the disease, but also the monitoring of efficacy of the therapies. Many "in vivo" imaging studies have been performed in order to better understand the neuronal circuits and the exact mechanisms involved in the principals MD, thanks in particular to the use of nuclear medicine techniques such as Single Photon Emission Computed Tomography (SPECT) or Positron Emission Tomography (PET), and the use of Magnetic Resonance (MR).

SPECT and PET are functional/anatomical nuclear medicine investigation that allows studying the brain circuits by the use of radiotracers (molecules combined with short half-life radioactive isotopes as $18 \mathrm{~F}$ or ${ }^{15} \mathrm{O}$, that decay emitting positrons). Nowadays the use of PET is combined with the use of Computed Tomography (PET/CT) or MR (PET/MR), integrating the functional data given by the radiotracers with the better anatomical information given by CT or MR. PET is considered the goldstandard imaging-technique for the study of the molecular expression on the surface of the cellular membrane (for example at synaptic level) or inside the cells, giving us useful information about potential neurochemical biomarkers involved in pathophysiology of psychiatric disorders [11]; PET is also able to study the metabolism of specific cerebral regions, calculating for example in the regional cerebral blood flow ( $\mathrm{rCBF})$, the cerebral oxygen metabolism rate $\left(\mathrm{CMRO}_{2}\right)$ or the cerebral glucose metabolism rate $\left(\mathrm{CMR}_{\mathrm{glu}}\right)$ by the use for example of ${ }^{18} \mathrm{~F}$-fluorodeoxyglucose $\left({ }^{18} \mathrm{FDG}\right)$ or ${ }^{15} \mathrm{O}$-water; the use of these investigations has been partially replaced by functional Magnetic Resonance Imaging (fMRI; see below) because of its higher spatial and temporal resolution and no ionizing radiations [11]. PET is very useful for the identification of potential biomarkers for target molecular therapies, in preclinical analysis for the analysis of dose-occupancy curves to estimate the correct dosage of antidepressant and/or antipsychotic and/or stabilizing mood drugs, and for the evaluation of the treatment effects [11].

MR is a non-invasive examination based on the use of radio waves and magnetic fields. In the study of psychiatric disorders it gives us not only anatomical information, i.e. structural Magnetic Resonance Imaging (sMRI) and Magnetic Resonance morphometry (MR-m), but can also give to the researchers functional information about CNS, using techniques such as Diffusion-Tensor Imaging (DTI), MR spectroscopy (MRs), and fMRI [6]. sMRI and MR-m helps in establishing and precisely quantifying the anatomical differences of the CNS structures between healthy controls (HCs) and ill patients, analysing parameters such as volume, morphology and gray/white matter ratio. 
The Diffusion Tensor Imaging (DTI) is a Diffusion Weighted Imaging (DWI) technique of investigation that measures the Brownian motions of the water molecules inside of the fibres of the white matter, i.e., the study of Fractional Anisotropy (FA); the motions of these water molecules are limited by the membranes of the white matter fibres. The images obtained show the course of the white matter fibres evidencing the maximum diffusivity of the water molecules along the white matter fibres (i.e. Fiber Tractography).

MRs studies the concentration of determined molecules such as choline or myo-inositol on a selected Region of Interest (ROI) of the CNS. Different approaches can be used for the evaluation of the neurochemical asset of a determined ROI, in particular $\left({ }^{1} \mathrm{H}\right)$ MRs and $\left({ }^{31} \mathrm{P}\right)$ MRs: the first one is important for the evaluation of the molecular concentration of chemical substances such as $\mathrm{N}$-acetyl aspartate (NAA), Myo-inositol (mIno), Creatine (Cr), lactate, amino-acids (glutamate/glutamine and GABA), Choline containing compounds (Cho), and the second one measures the products of cellular metabolism included Phosphocreatine (PCr), Adenosine Triphosphate (ATP), Phosphomonoester (PME) and Phosphodiester (PDE) (Table 1) [12].

\begin{tabular}{|l|l|}
\hline \multicolumn{2}{|c|}{ MRs techniques } \\
\hline \multicolumn{1}{|c|}{ (1H) MRs } & \multicolumn{1}{c|}{ (31P) MRs } \\
\hline N-acetyl aspartate (NAA) & Phosphocreatine (PCr) \\
\hline Myo-inositol (mIno) & Adenosine Triphosphate (ATP) \\
\hline Cretaine (Cr) & Phosphomonoester (PME) \\
\hline Choline containing compounds (Cho) & Phosphodiester (PDE) \\
\hline Lactate & \\
\hline Glutamate/Glutamine & \\
\hline GABA & \\
\hline Table 1: MRs techniques and main metabolites evaluable according to the technique of study
\end{tabular}

fMRI is a technique that provides an indirect measure of neuronal activity analysing the difference of the Blood Oxygen Level Dependent (BOLD) contrast of a specific ROI between two conditions (e.g., between rest and task execution), i.e., measuring the differences of the level of reduced and oxidized haemoglobin [12-14]. fMRI studies are performed using functional paradigms (for example showing on a monitor facial expressions, scenes or words), and evaluating the neuronal responses of the patients to these stimuli $[6,12,13,14]$.

In comparison with "post-mortem" pathological studies, the current biggest limit of the "in vivo" imaging studies for the evaluation of pure anatomical differences of neuronal circuits is their limited spatial resolution (e.g. $1 \mathrm{~mm}^{3}$ ) [12]. For a more detailed discussion about the technical aspects of these imaging modalities, we suggest the readers to read more specific publications. 


\section{Current evidences about the anatomical and functional findings in patients with MD.}

In order to better understand the current knowledge about the imaging markers of response to treatment, it is mandatory to analyse what we know up to date about the neural circuits involved MD, the differences between illness and HCs and between patients with different MD.

The CNS structures better studied in the pathophysiology of MD are the prefrontal cortex and the anterior limbic structures. We have already discussed about the fact that among the MD, BD and MDD are the most frequent and diagnosed in general population, and also the majority of the studies published in literature that investigated the pathologic mechanisms of MD are focused on these two entities. We will focus our attention separately on MDD and BD, underlying the main differences between these two conditions.

\section{Neural circuits involved in BD}

One of the main characteristics of $\mathrm{BD}$ is the deregulation in the emotional control along with cognition, accompanied by the recurrence of manic/hypomanic episodes and depressive episodes. Although there is still not general consensus about the conclusions of different studies, we can affirm that different "in vivo" imaging studies have evidenced that the principle CNS structures involved in the $\mathrm{BD}$ are located in prefrontal cortex (PFC), apparently involved in emotional regulation, and medial temporal and limbic structures (like the amygdala) involved in emotional memory formation $[6,11,13]$.

In the past nuclear imaging studies have been widely investigated the PFC and the limbic structures, although the results sometimes have been appeared controversial. One relatively recent study performed by the use of 18F-deoxyglucose-PET (18FDG-PET) in resting conditions evidenced a decreased metabolism in the dorso-lateral prefrontal cortex (DLPFC) and in the precuneus associated with an increased activity in the limbic/paralimbic regions in BD patients with acute mania [14]. Another 18FDG-PET study showed an increased metabolic activity of the left amygdala during depressive states in bipolar and unipolar patients compared to HCs, and this metabolic increased activity was correlated with increased plasmatic cortisol levels [15]. Several researches investigated also on the role of the serotonergic system, but the results have been inconsistent, although studies performed by the use of different radioligands investigated the role of multiple drugs acting on the expression of serotonine type $1 \mathrm{~A}(5 \mathrm{HT}-1 \mathrm{~A})$ and type 2 (5HT-2) receptors, serotonine transporters and synthesis, and on the regulation of the dopamine system (see below) [11]. 
Different sMRI and MRm studies have evidenced cortical thinning of the PFC in bipolar patients (BPs) at their first episode and of all ages $[16,17]$. Other researches showed enlarged amygdala and striatum volume, but not evidence of increasing neither decreasing volume in hippocampal volume $[18,19]$, and striatal enlargement has been also suspected to be a hereditable vulnerability factor for BD [20]. Other anomalies suspected to be associated with BD, especially in the paediatric population, are the increased volume of the supratentorial ventricles and a decreased sectional area of the corpus callosum [21]. Recent studies confirmed that patients with euthymic BD showed a reduced volume of the all subregions of the corpus callosum [22,23], posterior cingulum and prefrontal white matter [23] when compared with HCs. It has been also reported that the volume of the habenula (a structure of the human brain involved in several process, especially the stress responses and sleep/weak cycle) is significantly smaller in patients unmedicated BPs than in HCs [24]. A research by Radenbach et al. [25] evidenced that there are not significant differences in the volume of both the thalami between HCs and bipolar euthymic patients (treated or not). Recently many researches have investigated on the importance of the hippocampus in the pathogenesis of BD: in particular Otten M. et al. [26] in a recent extensive review demonstrated that in BPs the volume of hippocampal structures is reduced, and perhaps more pronounced in early-onset BPs. Also the signal of white matter was analysed, and it was demonstrated that BPs are more prone to show high signal intensity in white matter regions (but not in periventricular regions) compared to healthy population [27]. A mega-analysis performed in 2011 by Hallahan et al. [28] analysed the data of sMRI studies of eleven international groups, and found that bipolar subjects show an increased volume of the left temporal volume, and of the lateral ventricle and of the putamen on the right.

A few DTI researches that compared BPs with HCs showed significant regions of lower FA and abnormal elevated mean and radial diffusivity in particular in the white matter corpus callosum $[23,29]$; another study evidenced lower values of FA also in the prefrontal white matter [30]. These evidences can suggest an important role of the dysregulation of glial cells in the pathophysiology of the BD $[6,30]$.

MRs studies were performed in order to understand if in these CNS regions there is also a neurochemical difference between HCs and BPs. Different researches have shown for example that reduced levels of NAA were found in the PFC, in particular in the DLPFC [31], and in the left hippocampal regions (not in thalamus or putamen) in bipolar I patients [32] compared to the healthy population. A systemic analysis of different studies performed by Yildiz-Yesiloglu et al. [33] confirmed moreover increased levels of glutamate and glutamine in adult BPs compared to HCs and elevated $\mathrm{Cr} /$ Cho ratio in the basal nuclei of euthymic BPs. Another molecule investigated because of its relationship with lithium treatment in BPs is the mIno (see below): in fact it was demonstrated that in a children population affected by BD, compared to normal controls, the mIno levels in the Anterior Cingulate Cortex (ACC) were increased during maniac phase and decreased during depressive state [34]. 
Different fMRI studies, executed at rest or during manic or depressive states, confirmed the pivotal role of PFC and medial and temporal limbic structures in MD pathogenesis. Up to date, following the schemes of Strakowsky et al. [35], Philps et al. [36] and Brooks J0 $3^{\text {rd }}$ et al. [13], in order to explain the pathophysiology of BD, it has been adopted the so called "corticolimbic model", in which we can recognise three different circuits involved in regulation of internal and external emotional control (Scheme 1):

1. Ventrolateral circuit: this circuit is involved in the regulation and response to internally generated emotional states $[13,36]$. This circuit starts at the level the Ventro-Lateral Prefrontal Cortex (VLPFC); the signals are then sent to the ventromedial striatum, the globus pallidus, the thalamus and then back to the VLPFC. The VLPFC is also reciprocally connected with the anterior temporal cortex, BA 20 and 38.

2. Ventromedial circuit: this circuit is involved in the processing of external emotional stimuli $[13,37]$. It starts from the ventromedial prefrontal cortex (VMPFC), and continues through nucleus accumbens and the globus pallidus up to reach the thalamus; fibres that go back from the thalamus up to the VMPFC close the circuit. The amygdala is interconnected with the VMPFC directly and not directly by fibres that pass through the insula and the subgenual prefrontal cortex (SGPFC).

3. Anterior cingulate circuit: this circuit apparently is involved in the cognitive and affective processing [13]. It starts from ACC, and is connected to the thalamus, to the ventromedial striatum and the globus pallidus; the circuit is closed thanks to fibres that travel from the thalamus back to the ACC. The SGPFC send input to the ACC, amygdala and DLPFC (see below), and it is presumably involved in the integration of cognitive and emotional data $[13,18]$.

4. Dorsolateral circuit: this circuit connects the DLPFC, with the caudate nucleus, the globus pallidus and the thalamus, and receive input directly from ACC and SGPFC (see above).

The complexity of these circuits, and the uncountable connections present involved in the emotional and cognitive processes, make the study of BD really difficult; despite this fact, as we have evidenced before, the most important findings observed in BPs are the decreased activity of the PFC, which explain the cognitive and attention deficits observed, and the increased activity in the amygdala, responsible for the mood instability $[6,38,39,40,41]$.

\section{Neural circuits involved in MDD}

Different studies have showed various similarities in the CNS between patients affected by MDD and BD, also because they both manifest with depressive episodes [6]. Different researches 
found that the main structures involved in UD are the PFC, implied in an exaggerated response to negative emotions, and the brain stem structures such as locus coerleus and periacqueductal grey matter, responsible of different neuroendocrine alterations $[6,42]$.

Nuclear imaging studies using PET demonstrated reduction of the glucose metabolism in PFC [43], especially in the right side [44], as well as increased activity in the amygdala [15], similarly to what we have seen before about the depressive episode of BD. Other studies showed a pivotal role of the dopaminergic systems in the pathophysiology of MDD [11].

MRm studies demonstrated abnormalities characterized by loss of volume and reduced grey matter in the hippocampus/amygdala complex [44] and in the SGPFC [45,46]. The loss of volume of the hippocampus could be explained by a hyper-function of the hypothalamus-hypophysis-adrenal gland axis during stress periods, with increased cortisol production that conducts to an exaggerated glutammatergic release and the following apoptosis of neuronal cells [47]. The volume of the habenula in currently depressed women was significantly smaller than the healthy female controls [24]. The most significant difference between BD and MDD on MRm studies exposed in a review by Strakowski et al. [48] is the fact that when compared with healthy people the basal ganglia and amygdala appear smaller in MDD patients, and bigger in BD [18,47].

A DTI study indicated that naïve medication unipolar patients (UPs) show greater mean FA in the white matter of the Left Superior Longitudinal Fasciculus (LSLF) [49] and another study that compared HCs with patients UPs with melancholic and atypical features have shown in the second group decrease FA and increased mean diffusivity not only in the LSLF but also in the corpus callosum and in the inferior fronto-occipital fasciculus [50].

On MRs, most studies evidenced decreased levels of glutamate/glutamine and GABA in the CNS of UPs in comparison with HCs [51], in contrast with the increased glutamate levels evidenced in BPs [52]. No significant differences in levels of NAA, mIno and NAA/Cr rate have been evidenced between UPs and general population [51].

fMRI researches substantially confirm an important role of the PFC in the pathogenesis of MDD, in particular the disconnection between frontal and limbic structures [53], whereas studies on the activation of amygdala in response to the vision of sad (increased activity) and happy faces (decreased activity) did not show substantial differences between bipolar and unipolar patients in depressive state [13].

\section{E. Principal imaging markers of response to the treatment.}


We have just exposed the main information given by medical imaging researches on what we know about pathophysiological mechanisms which underlie the MD, and we have seen that we are still far away from a complete understanding of the exact pathophysiological mechanisms of these conditions. This is one of the reasons why in the introduction of this review we have underlined that at the moment in clinical practice there are still not standardized imaging markers useful for the diagnosis, the prognosis or the monitoring of treatment in patients with MD.

Despite these limitations, different studies performed by the use of MRI or Nuclear Medicine techniques have started to give us initial information about some potential marker of activity or predictive of functioning of drugs commonly used in the therapy of MD.

According to Hafenam DM et al. [54] the most robust evidences of psychotropic medications effects in BPs have been found in MR-m studies in comparison with both FMRI and DTI studies; moreover it has been seen that on imaging the effects of these medications are mainly "normalizing", i.e., the findings of CNS structures of illness patients after therapy and normal subjects are similar.

Now we will expose some of the main evidences founded in these studies according to the therapy (pharmacological or not) adopted. For every treatment cited below will be briefly explained the most known characteristics and indications; for more details about the mechanisms of action and/or technical execution we invite the readers to refer to specific publications.

\section{* Pharmacological therapy}

\section{Mood stabilizers: lithium and valproic acid}

The lithium salts have been used in psychiatry for the treatment of BD since the fifth decade of the twenty century; it is a mood-stabilizer, such as valproic acid, and it has not psychotropic effects on the healthy subjects [55]. The exact mechanism of action is still unknown, although there are many evidences that it has various molecular effects, in particular on the metabolism of the endogen monoamines, on the intracellular transduction of the signal, on the genetic expression and on the cellular survival, included an increased expression of neurotropic factor [55].

The valproic acid and divalproex (valproate semisodium) are drugs used for the treatment of epilepsy and BD; on the neurons it has similar effects to those of phenytoin and ethosuximide, inhibiting repetitive and prolonged depolarisations of the neural membranes in epileptic patients with a focused action on the $\mathrm{Na}^{+}$channels [56].

A few studies performed with nuclear medicine investigations investigated on the imaging of patients in treatment with these drugs. Nugent AC et al. [57] investigated the role of the lithium on the expression of 5HT-1A receptors in patients with BD using the [18F]trans-4-fluoro-N-(2-[4-(2methoxyphenyl)-piperazino]-ethyl)-N-(2-pyridyl)-cyclohexanecarboxamide ([ $\left.\left.{ }^{18} \mathrm{~F}\right] \mathrm{FCWAY}\right)$, and the mean 5HT-1A binding potential was significantly increased after the treatment with lithium or 
divalproex, in particular in the mesiotemporal cortex (hippocampus and amygdala). The effects of the treatment with valproate on the serotonin type $2 \mathrm{~A}$ (5HT-2A) receptors in acute manic patients was also investigated on PET using ${ }^{18} \mathrm{~F}$-setoperone, but the researchers have not found changes in $5 \mathrm{HT}-2 \mathrm{~A}$ receptor expression [58]. Other researches focalised their attention on the effect of these medications on the dopaminergic circuits: one PET study performed using 18F-6-fluoro-L-Dopa (18F-DOPA) evidenced that although the uptake of $18 \mathrm{~F}$-DOPA is not altered in acute manic patients when compared with HCs, after the therapy with divalproex sodium the presynaptic dopamine function was significantly lower [59]; another PET study realised with the use of ${ }^{11} \mathrm{C}$-raclopride evidenced not only that the dopamine D2-receptor density is not altered in nonpsychotic mania, but also that the treatment with divalproex sodium does not influence the D2-receptor availability [60].

sMRI and MR-m also have given important indications on the activity of mood stabilizers in BPs: in particular the researchers focused their attention on the volume changes of the hippocampal region (a CNS structure involved in the emotional and cognitive functions) following medical therapies with lithium and/or valproic acid. A study by Germana et al. [61] has shown that compared with other medications, bipolar patients treated with lithium (BPs-Li) showed incremented volume of the gray matter of the right SGPFC, of the post-central gyri, and of the left insula and hippocampus/amygdala complex. The neurotropic effects of lithium therapy were also confirmed by Bearden et al. [62] analysing the total hippocampus volume: in fact it was greater following lithium therapy compared with HCs (10.3\%) and unmedicated BPs (13.9\%). Similar results were found in another study conducted in a population-based twin cohort, revealing that the global hippocampal volume is significantly larger in BPs-Li than in HCs (9\%), non lithium-treated bipolar patients (BPs-NLi) (8\%) and non-bipolar co-twins (12\%), whereas the global volume of the hippocampal region was not different between non-lithium treated BPs, non-bipolar co-twins and control twins [63]. In adolescents with an age between 13 and 19 it was seen that the right hippocampal volume was larger in BPs-Li than HCs and this enlargement was not related to sex, even if in both the BPs and HCs cohorts the volume of this same region was larger in males than in the females [64]. Another research by Giakoumatos $\mathrm{CI}$ et al. [65] conducted with 3T MRI confirmed the previous data, revealing that BPs-NLi showed reduced cortical thickness and hippocampal volumes in comparison with HCs, whereas patients treated with lithium showed significant larger hippocampal volumes compared with BPs-NLi and not significant differences in cortical thickness and hippocampal volumes with the HCs; in another study it was moreover evidenced that the long-term therapy with lithium (2-4 years) is associated a bilateral increase in volume of the hippocampus, associated with some improvements in verbal memory performance [66]. The cortical thickening after lithium therapy in BPs was demonstrated also by Lyoo IK et al. [67], revealing that this increase reached the peak at the $10^{\text {th }}-12^{\text {th }}$ week of therapy, that was constant through 16 weeks of treatment, and that was also associated with positive clinical response; this finding has not been evidenced neither in BPs treated with valproic acid neither in HCs. Bearden CE et al. [68] in their study found that the cortical thickening of the gray matter of BPs-Li was 
in portions of the anterior limbic network, i.e., bilaterally at the level of the limbic and paralimbic cortex. In a study conducted by Savitz et al. [69] it was demonstrated not only that the lithium act on BPs inducing a cortical thickening, but also that the dimensions of both the amygdalae appeared increased in patients medicated with lithium or divalproex when compared with unmedicated BPs. It is remarkable to underlie that up to now the biggest review we have found in literature, conducted by Otten et al. [26], analized 25 different studies involving a total amount of 1043 patients and confirmed that lithium therapy can counteract the reduction in volume of hippocampal regions of BPs, whereas an international cooperative mega-analysis by Hallahan B. et al. [28] involving 321 BPs and 442 HCs confirmed that BPs-Li showed a significant increment of the hippocampal and amygdala volume when compared with BPs-NLi and HCs.

The lithium therapy determines also changes in the chemical equilibrium of CNS, and by now it seems clear from different MRs studies that the principal molecules involved in this process are mIno (involved in the intraneuronal phosphatidylinositol second messenger system, that seems to have a pivotal role in BD but not in other psychiatric diseases [70]) and glutamate/glutamine. In 2005 a review performed by Silverstone PH et al. [71] analized several animal and human MRs studies and they concluded that the levels of mIno were abnormal in particular in temporal and frontal lobes, in the basal ganglia and in the cingulate gyrus, but not in euthymic patients or HCs probably because of the effects of either lithium or valproate therapy. A research led by Friedman SD et al. [72] demonstrated that BPs-Li showed significant increased values of mIno and reduced values of glutamate/glutamine and GABA in the gray matter when compared with untreated HCs. A recent longitudinal study that analysed on MRs 24 BPs at baseline (week 0, drug-free patients) and after lithium monotherapy ( 6 weeks later) showed increased levels of glutamate/glutamine and NAA after therapy in the ACC, whereas the levels of mIno were increased in remitters patients and not in nonremitters patients [73]. A research by Patel NC et al. evidenced that the NAA levels are not influenced by lithium treatment in the VPFC of bipolar adult patients [74]. A lithium MRs study demonstrated moreover that cerebral lithium levels correlate with the serum levels, and that high brain lithium levels are correlated with frontal lobe dysfunction and elevated depression symptoms [75], whereas another lithium MRs study revealed that high brain lithium levels are also correlate with high levels of NAA and mIno [76].

fMRI has given new information about the functional changes of CNS circuits induced by the therapy with lithium and/or valproate. One research performed by Bell EC et al. [77] analysed the effects of lithium and valproate on CNS of healthy volunteers undergone to fMRI using a word generation, a spatial attention and a working memory task, before and after two weeks of therapy with lithium or valproate or placebo: the final results showed that the valproate group had decreased BOLD signal in the spatial attention and verbal tasks following therapy, whereas the lithium group had significative decreased BOLD signal also in the working memory task, suggesting different effects of these medications on the CNS structures. One of the first fMRI studies on bipolar subjects was 
conducted by Silverstone PH et al. [78], and analysed a group of 10 patients before and after two weeks of therapy ( 6 of these using lithium in monotherapy) using a word generation and a working memory task, and they found a significative reduced mean BOLD signal in Broca's area, supplemental motor area and the left pre-central gyrus, indicating that lithium may determine task- and statedependent effects on brain activation. Pavuluri MN et al. [79] examined the effects of risperidone (a not-conventional antipsychotic drug) plus placebo and divalproex plus placebo in a groug of paediatric BPs at baseline and after six weeks of therapy, and they found that the risperidone group showed an increased activation of the ventral striatum (rich in dopamine $\mathrm{D}_{2}$-receptors), in particular the left ventral striatum, and a greater activation of the left SGPFC and ACC in response to the vision of angry faces in comparison with the divalproex group; on the other hand, the divalproex group showed an enhanced activation of the fronto-temporal circuit relative to the risperidone group, in particular at the level of the left inferior frontal and right middle temporal gyri. Another CNS region studied on fMRI in paediatric BPs was the amygdala, and the group of patients responders to the therapy (one among divalproex, risperidone or lamotrigine) showed a greater connectivity of the amygdala with the frontolimbic network before and after the treatment when compared with the non-responders patients, suggesting a role of the amygdala as a potential responsivity biomarker for medication I bipolar patients [80].

Resuming, it is remarkable to say that even if several other studies have to be performed in order to better understand the imaging markers of response to mood stabilizers in $\mathrm{BD}$, the most investigated and robust finding is the effect of lithium on increasing the cortical thickness and the global volume of both the hippocampi and amygdalae on sMRI studies, and an increased of mIno concentrations on MRs.

\section{Antidepressant drugs.}

The effects on CNS of several antidepressant drugs have been investigated especially in the last years; a meta-analysis conducted by Delaveau P. et al. [81] that analysed different PET and MRI studies concluded that in patients affected by MDD following pharmacological treatment with antidepressants the activation of different cerebral regions was normalized, in particular the regions involved in the emotional processing: it was evidenced that those drugs determined an activation of the DLPFC, VLPFC and dorso-medial prefrontal cortex (DMPFC), and a decreased activation of amygdala, parahippocampal region, insula, hippocampus, ventral anterior cingulate and orbitofrontal cortices. Another review conducted by Höflich A. et al. [82] confirmed the fact that treatment with antidepressant drugs in MDD patients is able to partially restore the primarily altered processes of the CNS, even if not all of them; in particular it was underlined on SMRI how the volumetric differences between treated patients and HCs after medical therapy were reduced, on fMRI a normalization of the 
neural functioning of the CNS circuits, and on PET studies an influence the neuronal receptors asset (in particular the receptors for serotonin and noradrenaline).

In this paragraph our attention will be focalised on some studies that have investigated in particular on the role of two pivotal groups of antidepressant drugs, the so-called Selective Serotonin Reuptake Inhibitors (SSRI), and the Serotonine-Noradrenaline Reuptake Inhibitor (SNRI). As well as other categories of antidepressants, for example tricyclic antidepressants drugs (TCA), they are used in clinical practice mainly for the treatment of MDD, but they are also used for the treatment of other mood disorders and as a complementary tools for the control of the symptoms of different psychiatric conditions.

SSRI is a group of antidepressants derived from the TCA; their pharmacodynamics is still not clearly and completely understood, but it seems that these molecules act blocking selectively the reuptake of serotonin at a presynaptic level, with a series of molecular changes inducted by the greater availability of serotonin at the synaptic level [83]; among the principal drugs of these category we find paroxetine, fluoxetine, sertraline, citalopram, escitalopram. The SNRI drugs, as the name said, act inhibiting the reuptake of serotonin and noradrenaline, not interacting with muscarinic, histaminergic and adrenergic receptors [84]: among the principal drugs of this category it is remarkable to remember mirtazapine, duloxetine and venlafaxine.

Nuclear medicine researches analysed how the therapy with SSRI/SNRI can modify the molecular asset of the CNS circuits. A PET study by Lundberg J. et al. [85] used the radioligand $\left[{ }^{11} \mathrm{C}\right]$ MADAM in order to study the occupancy of the Serotonin Transporter (5-HTT) at the presynaptic level, comparing three groups of patients affected by MDD in remission, the first one treated with TCA, the second one with a SSRI and the third-one with mirtazapine: the group of patients in therapy with mirtazapine showed no occupancy of the 5-HTT receptors, whereas there were not significative differences in occupancy rates between the groups in therapy with SSRI and TCA, confirming a common mechanism of action of these two drugs on the 5-HTT. The 5-HTT occupancy rate in patients treated with SSRI has been also investigated by Ruhé et al. [86]: the researches evaluated a group of 15 patients affected by MDD in treatment with paroxetine 20-50 mg per day measuring the clinical response with the Hamilton Depression Rating Scale (HDRS), the 5-HTT occupancy rate in both amygdalae using SPECT examination using the radioligand [123I] $\beta$-CIT, and the amygdala activation in response to fearful and angry faces on fMRI, founding an association between positive clinical response to SSRI therapy, increased occupancy rate of 5-HTT, and reduction of activity of the left amygdala. The mechanisms of action of SSRI are evident not only on the 5-HTT, but also on the 5HT-1A receptor asset: a PET study by Lothe A. et al. [87] using [18F]MPPF has evidenced how after 30 days of treatment with SSRI in UPs the 5HT-1A receptors asset changed in the medial orbital regions of the cortex. A down regulation of the expression of 5HT-1A receptor expression has been also evidenced by another PET study conducted by Gray NA et al. [88] using a different radioligand, the [11C]WAY100635, in a group of UPs before and after 5-9 weeks of therapy with SSRI. The effect of SSRI and 
psychotherapy in MDD on the dopamine $\mathrm{D}(2 / 3)$ receptors asset was investigated by Hirvonen J. et al. [89]: two groups of UPs, one in treatment with fluoxetine and the other one with short-term psychodynamic psychotherapy, were evaluated on PET with the use of the radioligand $\left[{ }^{11} \mathrm{C}\right]$ raclopride, before and after 4 months of therapy, and the study showed that despite both treatments were clinically effective, only the group treated with fluoxetine showed an increased $\left[{ }^{11} \mathrm{C}\right]$ raclopride binding potential in the lateral thalamus that was not directly related with a clinical improvement.

The role of SSRI/SNRI in the therapy of MDD was also investigated using SMRI. Lai CH. et al [90] investigated on the changes on sMRI of subcortical structures in drug-naïve UPs in their first episode and panic disorder, finding that after 6 weeks of treatment with duloxetine the patients showed a modest volumetric increase of brainstem, left putamen, left hippocampus and bilaterally both the nuclei accumbens, that was also correlated with a response to the clinical symptoms. In another research conducted by Kong L. et al [91] 28 drug- naïve UPs at their first depressive episode were studied before and after treatment with fluoxetine, and compared with a group of 28 HCs, evaluating the gray matter volume (GMV): the non-medicated MDD patients showed a reduced gray matter volume in the right DLPFC, and in the left middle frontal gyrus in comparison with HCs, whereas the treated MDD patients showed greater GMV of the same above mentioned cortical regions when compared with HCs; a similar recent study led by Marano CM et al. [92] compared two group of patients, one constituted by unmedicated patients with diagnosis of Late-Life Depression (LLD) and the other one by HCs, founding that after a therapy of 12 weeks of citalopram the group of LLD patients showed increased GMV in the precuneus, in the superior and middle frontal gyri, in the cingulate gyrus and in the middle temporal gyrus, associated with mood, verbal memory and verbal fluency performances improvements.

Several fMRI studies have been made in order to evaluate the activity of SSRI/SNRI not only in UPs, but also in patients affected by other mood disorders. For example, Posner J et al. [93] studied the role of the duloxetine on the Default Mode Network (DMN; a network of CNS active when the subject is not focused on a goal-oriented activity) in patients with dysthymic disorder when compared with HCs, and they found that at baseline the coherence of activity of the DMN was greater in dysthymic patients in comparison with HCs, and that after 10 weeks of therapy the patients group showed a normalization of the DMN activity. Another study led by Fu CH et al. [94] investigated on the action of duloxetine in MDD patients comparing the data of SMRI and fMRI (resting state, emotional facial processing and negative attentional bias) at baseline and after 1, 8 and 12 weeks of treatment, and they found also in this case an increased activity of the anterior DMN in UPs after therapy. A fMRI research by Tao R. et al. [95] that compared a group of adolescent patients affected by MDD with HCs of the same age, analysing the brain activity during the vision of fearful and neutral expression faces revealed that the group of patients affected by MDD before the treatment with fluoxetine showed an exaggerated brain activation in response to the view of fearful relative to neutral faces of the CNS structures involved in the emotional process, included the amygdala, the subgenual anterior cingulate 
and the orbitofrontal cortices; the activity of these areas tended to normalize after 8 weeks of treatment with fluoxetine. As we have just seen, one of the principal mechanisms of action of the SSRI/SNRI drugs should be the indirect reduction of activity of amygdala; this ability has been well document by a series of fMRI studies, and the reduction of the activation of the amygdala has been proposed as a marker of functionality of antidepressant drugs. Ruhé et al. [96] demonstrated that after a 12 weeks treatment with paroxetine $20 \mathrm{mg}$ /day in MDD patients the activity of the amygdala in clinical responder UPs was reduced when compared to clinical non-responders. Another study this time led by Arnone D. et al. [97] monitored the brain activity of the amygdala of to groups of patients (one of UPs treated with citalopram and the other one constituted by HCs) on fMRI during an indirect face-emotion processing task, and in conclusion they found that after 8 weeks of treatment with citalopram the UPs had a reduction of the abnormal amygdala hyper-activation in response to sad but not fearful faces. Two different fMRI studies monitored the action of escitalopram on the activity of amygdala: the first study led by Godlewska BR et al. [98] confirmed that in UPs a short-term SSRI treatment is able to decrease the activation of amygdala prior to clinical improvements; the second one, led by Chen YT et al. [99] demonstred that although in every UP there is a lateralized amygdala activity with the predominance of the left or right side and that after the treatment this asymmetry persisted but the difference of activity between the two amygdalae was greater, and also that the variation of activity was greater in the left amygdala. It has been also demonstrated that in patients with MDD the treatment with escitalopram is able to modulate the resting-state brain activity of the fronto-limbic circuits [100], to increase and normalize the activation of the ventral striatum during incentive cue processing [101], and to reduce and normalize the activity of the DMPFC (in correlation with symptomatic improvements) [102]. One research conducted by Yang R. et al. [103] demonstrated that patients who suffered of MDD after 8 weeks of treatment with sertraline showed an increased connectivity between the hypothalamus and several other CNS structures, comprised the claustrum, the putamen, the caudate, the insula and the dorsolateral prefrontal, orbitofrontal and anterior cingulate cortices. Finally one DTI study of Korgaonkar MS et al. [104] analysed a group of MDD patients at baseline and after 8 weeks treatment with escitalopram or sertraline or venlafaxine showed that altered connectivity of cingulum cingulate and stria terminalis tracts are predictive of remission of outcome to treatment with an accuracy of 62 .

In conclusion, it is remarkable to underlie that up to date the most relevant imaging findings related to the action of SSRI on CNS are represented by occupancy of 5-HTT transporters at the presynaptic level, the down-regulation of the expression of 5HT-1A receptors, by a normalization of the activity of the DMN, and by the reduction of the hyperfunction of the both amygdalae (in particular the left one) evidenced on PET and fMRI.

\section{Ketamine}


Ketamine is an anaesthetic drug that acts as antagonist of the NMDA receptor for glutamate, also considered an abuse substance [105,106]; its use has been tested in UPs not responsive to the conventional therapies. Its effect on CNS structures was investigated on PET by Carlson PJ et al. [107] and they analyzed the glucose metabolism rate of 20 unmedicated patients before and immediately after a single dose of ketamine intravenous infusion $(0.5 \mathrm{mg} / \mathrm{kg}$ over 40 minutes), finding that the rapid antidepressant response after treatment was associated with a decreased metabolic activity on the right hemisphere in the ventrolateral and prefrontal cortices, in the habenula and in the insula.

Abdallah CG et al. [108] conducted a sMRI analysis of the brain of medication-free UPs before they underwent on a treatment with ketamine infusion $(0.5 \mathrm{mg} / \mathrm{kg}$ over 40 minutes) and they suggested that ketamine is able to exert antidepressive effects on patients with a baseline relatively small hippocampus.

A MRs study conducted on healthy volunteers by Taylor MJ et al. [109] found that before, during and after the intravenous infusion of ketamine at $0.5 \mathrm{mg} / \mathrm{kg}$ over 40 minutes there was no visible effect on the concentration of glutamate and glutamine in the anterior cingulate cortex despite his effects on mental state, whereas in another MRs study by Milak MS et al. [110] it has been evidenced that in MDD patients treated with intravenous infusion of ketamine $(0.5 \mathrm{mg} / \mathrm{kg})$ there was an increased concentration of the levels of glutamate/glutamine and GABA in the medial prefrontal cortex, and the peak of concentration was reached above about 26 minutes after the starting of the drug-infusion.

Murrough JW et al. [111] evaluated on fMRI the effects of a single infusion of ketamine $(0.5$ $\mathrm{mg} / \mathrm{kg}$ ) on 20 treatment resistant MDD patients using a visual task with happy and sad faces before and after treatment, comparing the data with 20 HCs that did not receive any treatment, and they found that at baseline treatment resistant MDD patients showed reduced neural activity in the right caudate to the vision of happy faces when compared with HCs, but following therapy with ketamine the neural response at this level was significantly higher.

\section{* Non-Pharmacological therapy}

\section{Electroconvulsive Therapy}

The Electroconvulsive Therapy (ECT) is a non-pharmacological therapy used in case of severe and/or treatment (pharmacological and psychoterapeutical) resistant UPs or BPs. It usually gives clinical results after 6-8 applications, and the major adverse collateral effect usually is a transient memory deficit [112].

Nuclear medicine investigations focused on the role of the receptor asset of CNS structures after ECT. Saijo T. et al [113] performed a PET study with the use of radioligand [11C]FLB 457 that compared a population of eleven HCs with seven UPs before and after 6-7 ECT treatment in order to evaluate the $D_{2}$ receptor binding potential, and they found that after the ECT therapy all the patients 
showed clinical improvement and reduced $\mathrm{D}_{2}$ receptor binding potential in the rostral anterior cingulate. The same team, in another PET study [114] analysed a group of UPs with the use of $\left[{ }^{11} \mathrm{C}\right]$ WAY 100635 and found that there was not significant difference in 5HT-1A receptor binding between pre- and post 6-7 sessions of ECT; on the other hand Lanzemberger et al. [115] in a more recent study that analysed 12 UPs on PET with the use of [carbonyl-11C ] WAY 100635 radioligand found that after an average of 10 sessions of ECT in all the cortical and subcortical regions of the brain (except the occipital cortex and cerebellum) there was a significant decrease of the 5HT-1A binding potential. The role of ECT in the expression of 5HT-2 receptors was investigated on PET by Yatham LN et al. [116] with the use of [18F]setoperone in a population of 15 drug-resistant UPs who underwent on PET examination after ECT therapy performed on a drug-free washout period, and they found that the number of 5HT-2 receptors was reduced in all the cortical areas, mainly in the right hemisphere.

Several SMRI researches investigated the volume changes of the main CNS structures after ECT. Ota M. et al. [117] evaluated the gray matter volumes in a group of 15 UPs in treatment with antidepressants before and after ECT, and they found that after ECT there was a significant increase of volume of both medial temporal lobes; the group of Bouckaert F et al. [118] obtain the same results after right unilateral ECT therapy in a population of 28 patients with late life depression, but they noticed also an increased volume of the caudate nuclei, insulae and posterior superior temporal regions, more pronounced on the side of the stimulation. Tendolkar I et al. [119] also found increased volume of both hippocampi and amygdale with a preserved global cerebral volume in a group of 15 antidepressant free MDD patients, and Ten Doesschate F et al. [120] found not only that a larger amygdala pre-treatent volume is predictive of a better outcome after ECT therapy independently from other factors, but also that the left pre-treatment amygdala volume had greater predictive value in comparison with the contralateral. A study by Nordanskog P et al. [121] analysed 12 patients affected by MDD at baseline, immediately after ECT treatment, and after 6 and 12 months, and they found that the volume increase of hippocampi was immediate after ECT, but disappeared in the first MRI followup after 6 months, and moreover was not related to clinical or cognitive outcome.

The role of hippocampus have been studied not only on SMRI but also on fMRI by Abbott CC et al. [122] and they found that after ECT treatment in UPs the right hippocampal connectivity increased and normalized (when compared with HCs), in correlation also with good clinical outcomes and with increased volumes of the right subiculum regions and right dentate gyrus and bilaterally both the hippocampal cornu ammoni, even if these results could have been influenced by the stimulus delivery method (predominantly on the right side). Cano $\mathrm{M}$ et al. [123] noted that in treatment-resistant MDD, the ECT determines different changes in the brain, in particular a reduction of activity within the limbic circuit and an increase in the limbic-prefrontal circuits. Another study by Perrin JS et al [124] documented that clinical improvements observed in 9 UPs after ECT were related with a decrease of the activity of the DLPFC. Liu Y et al. [125] analysed on fMRI 23 treatment-naïve UPs before and after ECT and they observed that the left subgenual anterior cortex activity increased at the end of the 
therapy, and this activity increasing was related with clinical improvements. Finally, a study by Beall et al. [126] performed on fMRI during working memory and affective tasks in a group of 6 ECT-naïve patients before and after treatment revealed that the clinical remission was associated with an increased of connectivity of the right DLPFC and posterior cingulate cortices, and a decreased activity of the orbitofrontal cortex.

We can summarize that the most robust evidences of the action of ECT on the CNS circuits are the reduced expression of 5HT-2A receptors, the bilateral volumetric increase of temporal lobes (in particular the hippocampus), and the reduction of activity of the DLPFC in a contest of global deactivaction of the limbic-prefrontal circuits.

\section{Transcranial Magnetic Stimulation}

The Transcranial Magnetic Stimulation (TMS) is an experimental non-pharmacological therapy used in particular in case of treatment resistant MDD [112]. We expose a few studies that have started to analyse the effect of this therapy.

One PET research performed by Baeken C. et al. [127] studied a group of 21 antidepressantfree and resistant to treatment UPs before and after a cycle of High-Frequency Repetitive TMS (HFRTMS) of the left DLPFC, and they found that before the treatment higher metabolic activity in the ACC and DLPFC were associated with a better clinical remission, whereas after the treatment metabolic changes where observed in particular at the level of BA 24 and 32 in the ACC.

One sMRI by Furtado CP et al. [128] tried to identify on sMRI potential predictors of response to repetitive TMS in patients with treatment resistant depression, and they found that there were not significant differences in medial temporal lobes pre-treatment volume between responders and nonresponder subjects, whereas a smaller pre-treatment volume of the left hippocampus was correlated with a better subjective clinical outcome.

Finally, Hernández-Ribas R et al. [129] found that UPs with smaller deactivations of the anterior cingulate, left medial orbitofrontal and right middle frontal cortices, and greater activation of the left ventral-caudal putamen where associated with a better clinical outcome after a cycle of repetitive TMS of the DLPFC.

\section{Psychotherapy}

There are different kind of psychotherapy used in the treatment of MDD and BD, but they are usually associated with pharmacological treatments [112]. The most used types of psychotherapy in clinical practice are the Cognitive-Behavioural Psychotherapy (CBP) that generally takes place in 1525 weekly individual sessions in order to modify the cognitive dysfunction at the base of the MDD, and the Interpersonal Psychotheray (IP), that generally takes place in 12-16 weekly sessions and try to 
identify and modify those interpersonal situations which determine and contribute to maintain the depressive state [112]. For BD patients it is also indicated a short-term (3-4 months of weekly meetings) psycho-educative treatment, usually for group of patients and their relatives, in order to understand the disease and know how to manage the problems related with that, included for example the recognition and management of the stressful events that could give origin to a crisis [112].

As discussed before, psychotherapy in MD is a treatment usually performed in association with pharmacological therapy, and for this reason up to date it is hard to find studies that analysed the effects of this kind of treatment alone.

One sMRI study performed by Mackin RS et al. [130] tried to correlate the impact of cortical thickness on the therapeutic response to psychotherapy in patients affected by LLD and they found that all the patients studied, in comparison with HCs, showed thinner cortex in right frontal, temporal and parietal regions, and after a cycle of psychotherapy responder patients (50\%), when compared to treatment responders, showed thicker cortex at various levels, in particular the posterior cingulate and parahippocampal corteces bilaterally, on the left the paracentral, the cuneus and precuneus and the insular corteces, and on the right the medial orbitofrontal and the lateral occipital cortices; the findings suggested that a reduced bilateral cortical thickness should be considered as a useful biomarker of response to psychotherapy.

Different fMRI researches have been conducted in order to understand the role of psychotherapy on the brain networks. Garrett AS et al. [131] analysed two groups of youth subjects, one that consisted of 12 patients between 13 and 17 years old with symptoms of depression and/or mania, and the other one constituted of $12 \mathrm{HCs}$, and they found that the group of youth patients showed a reduced activation of the DLPFC and an increased activation of the posterior cingulate cortex when compared with the HCs, whereas after treatment they showed a decreased activity of the amygdala and an increased activity of the DLPFC (this one associated with an improvement of the mania symptoms). Another study by Buchheim A. et al [132] compared a group of 17 HCs with 16 recurrently medication-naïve UPs patients, before and after 15 months of psychodynamic psychotherapy, finding that after the therapy the patients showed a reduction of the activity of the left anterior hippocampus and amygdala, subgenual cingulate and medial prefrontal cortex, associated with a general improvement of the depressive mood, whereas the decreased activation of the medial prefrontal cortex was associated with a general symptom improvement. In a research conducted by Ives-Deliperi et al. [133] 23 BPs underwent neuropsychological testing and fMRI and of these 16 received a cycle of 8 weeks treatment with mindfulness-based cognitive therapy and the other 7 were put in the waiting-list: the fMRI of control executed at the end of the observational period in both the groups revealed increased activation of the medial prefrontal and posterior parietal lobe cortices associated with clinical improvement of the symptoms, in particular of the anxiety and emotional regulation in the group who underwent to mindfulness-based cognitive psychotherapy. The effect of the cognitive-behavioural psychotherapy on the brain networks have been investigated by Straub J et 
al. [134]: they analysed 22 unmedicated MDD adolescents divided in two groups, one that underwent on five sessions of cognitive-behavioural psychotherapy and the other one put in the waiting list, and all of them have been analysed before and after the end of the treatment of the group who underwent to the psychotherapy, finding that the reduction of the clinical symptoms in the treated group was related also with a changed activity in the left amygdala and hippocampus and bilaterally in the subgenual anterior cingulate cortex. Klein JP et al. [135] analysed the effects of a 12-weeks specialised psychotherapy on a group of 10 MDD patients, at baseline and after the treatment, on fMRI during the visualization of faces with positive and negative expression, and they found that the $60 \%$ of the patients responded to the treatment and showed increased activity of the left amygdala after the treatment. The effects of guided imagery psychotherapy have been investigated by Huang $\mathrm{X}$ et al. [136], founding that in UPs who underwent to 5 weeks of guided imagery a higher pre-treatment regional homogeneity in the dorsal anterior cingulate gyrus was associated with a better clinical response. Recently Yuan $\mathrm{H}$ et al. [137] have investigated on a new technique called "real-time functional magnetic resonance imaging neurofeedback" (rtfMRI-nf) expected to upregulate the amygdalar activity during recall of happy autobiographical memories in UPs, adopting this technique in two groups of patients, one constituted by 27 UPs, and the other one by 27 HCs, finding that in MDD the abnormal hypoconnectivity of the left amygdala was reversed after rtfMRI-nf, and this was in association with an improvement of the depressive symptoms.

\section{F. Conclusion}

As we have seen, the topic of this review was to analyse some of the main studies conducted up to date in order to evaluate the most important imaging findings related to the action of the pharmacological and non-pharmacological treatments in mental disorders. Unfortunately up to now there are not many available clinical imaging studies that analyse the functional and anatomical changes of the CNS induced by the therapies, with a lack of convergence of the results. Anyway some stronger evidences on the impact of the therapies for mood disorders on the anatomy and function of the brain are available for mood stabilizers, antidepressant drugs and the electroconvulsive therapy.

The major limitation of this analysis is that the data collected derived from researches that have enrolled few patients, and of curse by the fact that we are still far away from a complete comprehension of the aetiology and pathophysiological mechanisms at the base of mental disorders. New researches supported by further technological improvements in imaging techniques, especially the development of fMRI and new radiodrugs in nuclear medicine investigations, will help us to better define the anatomical and functional pathological findings of mood disorders and then to identify of standardized imaging markers for the evaluation of the treatment response. 


\section{References}

1. ESEMeD/MHEDEA 2000 Investigators, European Study of the Epidemiology of Mental Disorders (ESEMeD) Project. - Use of mental health services in Europe: results from the European Study of the Epidemiology of Mental Disorders (ESEMeD) project. - Acta Psychiatr Scand 2004: 109 (Suppl. 420): 47-54

2. Kessler RC et al. - Lifetime prevalence and age-of-onset distributions of DSM-IV disorders in the National Comorbidity Survey Replication. Arch Gen Psychiatry. 2005; 62(6):593-602

3. Kessler RC et al. - Prevalence, Severity, and Comorbidity of 12-Month DSM-IV Disorders in the National Comorbidity Survey Replication - Arch Gen Psychiatry. 2005; 62(6):617-627

4. Phillips MR et al. Prevalence, treatment, and associated disability of mental disorders in four provinces in China during 2001-05: an epidemiological survey. Lancet. 2009; 373 (9680):2041-53.

5. World Health Organization. The world health report 2001: mental health - new understanding, new hope. Geneva, Switzerland: World Health Organization, 2001.

6. Agarwal et al . - Update on the use of MR for assessment and diagnosis of psychiatric diseases Radiology. 2010 Apr;255(1):23-41

7. American Psychiatric Association. Diagnostic and Statistical Manual of Mental Disorders, 5th ed. Arlington: American Psychiatric Association, 2013.

8. American Psychiatric Association. Diagnostic and statistical manual of mental disorders, 4th ed. Washington: American Psychiatric Association, 1994.

9. Regier Darrel A. et al. - The DSM-5: classification and criteria changes - World Psychiatry 2013;12:92-98

10. Goldberg DP, Krueger RF, Andrews G et al. Emotional disorders: cluster 4 of the proposed meta-structure for DSM-V and ICD-11. Psychol Med 2009;39:2043-59.

11. Qiaozhen C. et al. - Molecular imaging in patients with mood disorders: a review of PET findings - Eur J Nucl Med Mol Imaging (2011) 38:1367-1380

12. Strakowski SM - The functional neuroanatomy of bipolar disorder: a review of neuroimaging findings - Mol Psychiatry. 2005 Jan;10(1):105-16

13. Brooks JO 3rd et al. - Diagnostic and clinical implications of functional neuroimaging in bipolar disorder - J Psychiatr Res. 2014 Oct;57:12-25

14. Brooks III JO et al. - Metabolic evidence of corticolimbic dysregulation in bipolar mania. Psychiatry Res. 2010;181(2):136-40.

15. Drevets WC et al. - Glucose metabolism in the amygdala in depression: relationship to diagnostic subtype and plasma cortisol levels. Pharmacol Biochem Behav. 2002;71(3):431-47.

16. Wilke $\mathrm{M}$ et al. - Voxel-based morphometry in adolescents with bipolar disorder: first results. Psychiatry Res 2004;131 ( 1 ): 57 - 69 .

17. Lyoo IK et al - Regional cerebral cortical thinning in bipolar disorder . Bipolar Disord 2006; 8 ( 1 ): 65 - 74.

18. Strakowski SM et al. - The functional neuroanatomy of bipolar disorder: a review of neuroimaging findings. Mol Psychiatry 2005; 10 ( 1 ): 105 - 116 .

19. Rosso et al. - Reduced amygdala volumes in first episode bipolar disorder and correlation with cerebral white matter . Biol Psychiatry 2007; 61 ( 6 ): 743 - 749.

20. Strakowski SM et al. - Ventricular and periventricular structural volumes in fi rst- versus multipleepisode bipolar disorder . Am J Psychiatry 2002 ; 159 ( 11 ): 1841 - 1847.

21. Lyoo IK et al. - White matter hyperintensities on magnetic resonance imaging of the brain in children with psychiatric disorders. Compr Psychiatry 2002 ; 43 ( 5 ): 361 - 368.

22. Lloyd AJ et al. - Corpus callosum changes in euthymic bipolar affective disorder - Br J Psychiatry. 2014 Feb;204(2):129-36.

23. Emsell L. et al. - White matter differences in euthymic bipolar I disorder: a combined magnetic resonance imaging and diffusion tensor imaging voxel-based study. - Bipolar Disord. 2013 Jun;15(4):365-76.

24. Savitz JB et al. - Habenula volume in bipolar disorder and major depressive disorder: a highresolution magnetic resonanceimaging study. - Biol Psychiatry. 2011 Feb 15;69(4):336-43

25. Radenbach $\mathrm{K}$ et al. - Thalamic volumes in patients with bipolar disorder. - Eur Arch Psychiatry Clin Neurosci. 2010 Dec;260(8):601-7

26. Otten M. et al. - Hippocampal structure and function in individuals with bipolar disorder: a systematic review - J Affect Disord. 2015 Mar 15;174:113-25. 
27. Kempton MJ et al. - Meta-analysis, database, and meta-regression of 98 structural imaging studies in bipolar disorder . Arch Gen Psychiatry 2008 ; 65 ( 9 ): 1017 - 1032.

28. Hallahan B et al. - Structural magnetic resonance imaging in bipolar disorder: an international collaborative mega-analysis of individual adult patient data - Biol Psychiatry. 2011 Feb 15;69(4):326-35.

29. Barysheva $\mathrm{M}$ et al. - White matter microstructural abnormalities in bipolar disorder: A whole brain diffusion tensor imaging study - Neuroimage Clin. 2013 Apr 5;2:558-68

30. Adler CM et al. - Abnormal frontal white matter tracts in bipolar disorder: a diffusion tensor imaging study Bipolar Disord. 2004 Jun;6(3):197-203

31. Winsberg ME et al. - Decreased dorsolateral prefrontal N-acetyl aspartate in bipolar disorder - Biol Psychiatry. 2000 Mar 15;47(6):475-81.

32. Scherk H et al. - Neurochemical pathology in hippocampus in euthymic patients with bipolar I disorder . Acta Psychiatr Scand 2008 ; 117 ( 4 ): 283 - 288.

33. Yildiz-Yesiloglu A et al. -. Neurochemical alterations of the brain in bipolar disorder and their implications for pathophysiology: a systematic review of the in vivo proton magnetic resonance spectroscopy findings . Prog Neuropsychopharmacol Biol Psychiatry 2006 ; 30 ( 6 ): 969 - 995.

34. Davanzo P.et al. - Decreased anterior cingulate myo-inositol/creatine spectroscopy resonance with lithium treatment in children with bipolar disorder . Neuropsychopharmacology 2001; 24 ( 4 ): 359 - 369.

35. Strakowski SM et al. - The functional neuroanatomy of bipolar disorder: a consensus model. Bipolar Disord 2012;14:313e25.

36. Phillips ML, Swartz HA. A critical appraisal of neuroimaging studies of bipolar disorder: toward a new conceptualization of underlying neural circuitry and a road map for future research. Am J Psychiatry 2014.

37. Phillips ML et al. - A neural model of voluntary and automatic emotion regulation: implications for understanding the pathophysiology and neurodevelopment of bipolar disorder. Mol Psychiatry 2008;13:829e833e57.

38. Frangou S. - Functional neuroimaging in mood disorders - Psychiatry Volume 5, Issue 5, 176-179

39. Yurgelun-Todd DA et al. - fMRI during affect discrimination in bipolar affective disorder. Bipolar Disord 2000; 2 ( 3 pt 2 ): $237-248$.

40. Malhi GS et al. - An emotional Stroop functional MRI study of euthymic bipolar disorder. Bipolar Disord 2005; 7 ( suppl 5 ): 58 - 69.

41. Foland LC et al. - Evidence for defi cient modulation of amygdala response by prefrontal cortex in bipolar mania. Psychiatry Res 2008; 162 ( 1 ): 27 - 37.

42. Drevets et al. - Brain structural and functional abnormalities in mood disorders: implications for neurocircuitry models of depression . Brain Struct Funct 2008; 213 ( 1-2 ): $93-118$.

43. Baxter L. R. et al. - Reduction of prefrontal cortex glucose metabolism common to three types of depression. Arch Gen Psychiatry. 1989 Mar;46(3):243-50.

44. Dunn RT, et al. - Principal components of the Beck Depression Inventory and regional cerebral metabolism in unipolar and bipolar depression. Biol Psychiatry 2002; 51: 387-99.

45. Beyer JL et al. - Volumetric brain imaging fi ndings in mood disorders. Bipolar Disord 2002; 4 ( 2 ): 89 - 104.

46. Drevets et al. - Subgenual prefrontal cortex abnormalities in mood disorders. Nature 1997; 386 (6627 ): 824 827.

47. Lee AL et al. - Stress and depression: possible links to neuron death in the hippocampus. Bipolar Disord 2002; 4 ( 2 ): $117-128$.

48. Strakowski SM - Volumetric MRI studies of mood disorders: do they distinguish unipolar and bipolar disorder? Bipolar Disord 2002; 4 ( 2 ): 80 - 88.

49. Wang $Y$ et al. - White matter abnormalities in medication-naïve adult patients with major depressive disorder: tract-based spatial statistical analysis. - Neuro Endocrinol Lett. 2014;35(8):697-702.

50. Ota M. et al. - White matter abnormalities in major depressive disorder with melancholic and atypical features: A diffusion tensor imaging study. - Psychiatry Clin Neurosci. 2015 Jun;69(6):360-8

51. Lee TS et al. - Molecular Imaging for Depressive Disorders - AJNR Am J Neuroradiol. 2014 Jun;35(6 Suppl):S44-54

52. Yildiz-Yesiloglu A et al. - Review of ${ }^{1} \mathrm{H}$ magnetic resonance spectroscopy findings in major depressive disorder: a meta-analysis. Psychiatry Res 2006; 147 (1): 1 - 25.

53. Taylor et al. - Frontal white matter anisotropy and antidepressant remission in late-life depression. PLoS One 2008; 3 ( 9 ): e3267

54. Hafeman DM et al. - Effects of medication on neuroimaging findings in bipolar disorder: an updated review. Bipolar Disord. 2012 Jun;14(4):375-410.

55. Baldessarini R.J. et al. - Farmacoterapia delle psicosi e della mania - Brunton L.L. et al. - Goodman and Gilman’s The pharmacological Basis of Therapeutics, $11^{\circ}$ edition - Italian edition - McGrawHill - Chapter 18 - pp 461-500.

56. McNamara JO - Farmaci per la terapia delle epilessie - Brunton L.L. et al. - Goodman and Gilman's The pharmacological Basis of Therapeutics, $11^{\circ}$ edition - Italian Edition - McGrawHill - Chapter 19 - pp 501 525. 
57. Nugent AC et al. - Mood stabilizer treatment increases serotonin type 1A receptor binding in bipolar depression - J Psychopharmacol. 2013 Oct;27(10):894-902.

58. Yatham LN et al. - A positron emission tomography study of the effects of treatment with valproate on brain 5-HT2A receptors in acute mania - Bipolar Disord. 2005;7 Suppl 5:53-7.

59. Yatham LN et al. - PET study of [(18)F]6-fluoro-L-dopa uptake in neuroleptic- and mood-stabilizer-naive first-episode nonpsychotic mania: effects of treatment with divalproex sodium - Am J Psychiatry. 2002 May;159(5):768-74.

60. Yatham LN et al. - PET study of the effects of valproate on dopamine $\mathrm{D}(2)$ receptors in neuroleptic- and mood-stabilizer-naive patients with nonpsychotic mania - Am J Psychiatry. 2002 Oct;159(10):1718-23

61. Germana C. et al. - The effects of lithium and anticonvulsivants on brain structure in bipolar disorder - Acta Psychiatr Scand 2010 Dec; 122(6):481-7

62. Bearden et al. - Three-dimensional mapping of hippocampal anatomy in unmedicated and lithium-treated patients with bipolar disorder - Neuropsychopharmacology 2008 May;33(6): 1229-38.

63. Van Erp TG et al. - Hippocampal morphology in lithium and non-lithum-treated bipolar I disorder patients, non-bipolar co-twins, and control twins - Hum Brain Mapp 2012 Mar;33(3):501-10.

64. Baykara B. et al. - Increased Hippocampal volumes in lithium treated adolescents with bipolar disorders: a structural MRI study. - J Affect Disord 2012 May;138(3):433-9.

65. Giakoumatos CI et al. - Effects of lithium on cortical thickness and hippocampal subfield volumes in psychotic bipolar disorder - J Psychiatr Res. 2015 Feb;61:180-7.

66. Yucel K. Et al. - Bilateral hippocampal volume increases after long-term lithium treatment in patients with bipolar disorder: a longitudinal MRI study - Psychopharmacology (Berl). 2007 Dec;195(3):357-67.

67. Lyoo IK et al. - Lithium-induced gray matter volume increase as a neural correlate of treatment response in bipolar disorder: a longitudinal brain imaging study.

68. Bearden CE et al. - Greater cortical gray matter density in lithium-treated patients with bipolar disorder - Biol Psychiatry. 2007 Jul 1;62(1):7-16.

69. Savitz J. Et al. - Amygdala volume in depressed patients with bipolar disorder assessed using high resolution 3T MRI: the impact of medication - Neuroimage 2010 Feb 15;49(4):2966-76.

70. Kim H. et al. - A review of the possible relevance of inositol and the phosphatidylinositol second messenger system (PI-cycle) to psychiatric disorders--focus on magnetic resonance spectroscopy (MRS) studies - Hum Psychopharmacol. 2005 Jul;20(5):309-26

71. Silverstone $\mathrm{PH}$ et al. - Bipolar disorder and myo-inositol: a review of the magnetic resonance spectroscopy findings - Bipolar Disord. 2005 Feb;7(1):1-10

72. Friedman SD et al. - Lithium and valproic acid treatment effects on brain chemistry in bipolar disorder - Biol Psychiatry. 2004 Sep 1;56(5):340-8.

73. Machado-Vieira R. et al. - A Longitudinal (6-week) 3T 1H-MRS Study on the Effects of Lithium Treatment on Anterior Cingulate Cortex Metabolites in Bipolar Depression - Eur Neuropsychopharmacol. 2015 Sep 25. pii: S0924-977X(15)00279-5.

74. Patel NC et al. - Temporal change in N-acetyl-aspartate concentrations in adolescents with bipolar depression treated with lithium - J Child Adolesc Psychopharmacol. 2008 Apr;18(2):132-9

75. Forester BP et al. - Brain lithium levels and effects on cognition and mood in geriatric bipolar disorder: a lithium-7 magnetic resonance spectroscopy study - Am J Geriatr Psychiatry. 2009 Jan;17(1):13-23.

76. Forester BP. et al. - Brain lithium, N-acetyl aspartate and myo-inositol levels in older adults with bipolar disorder treated with lithium: a lithium-7 and proton magnetic resonance spectroscopy study - Bipolar Disord. 2008 Sep;10(6):691-700.

77. Bell EC et al. - Differential effects of chronic lithium and valproate on brain activation in healthy volunteers. - Hum Psychopharmacol. 2005 Aug;20(6): 415-24.

78. Silverstone $\mathrm{PH}$ et al. - Lithium alters brain activation in bipolar disorder in a task - and state- dependet manne: an fMRI study. - Ann Gen Psychiatry. 2005 Jul 19;4:14.

79. Pavuluri MN et al. - Risperidone and divalproex differentially engage the fronto-striato-temporal circuitry in pediatric mania: a pharmacological functional magnetic resonance imaging study. - J Am Acad Child Adolesc Psychiatry. 2012 Feb;51(2): 157-170.e5.

80. Wegbreit E. et al. - Amygdala functional connectivity predicts pharmacotherapy outcome in pediatric bipolar disorder. - Brain Connect. 2011;1(5):411.22

81. Delaveau P. et al. - Brain effects of antidepressants in major depression: a meta-analysis of emotional processing studies - J Affect Disord. 2011 Apr;130(1-2):66-74.

82. Höflich A. et al. - Imaging treatment effects in depression - Rev. Neurosci. 2012;23(3):227-52

83. Ross J. Baldessarini - Farmacoterapia della depressione e dei disturbi d'ansia - Brunton L.L. et al. - Goodman and Gilman's The pharmacological Basis of Therapeutics, $11^{\circ}$ edition - Italian Edition - McGrawHill Chapter 17 - pp 429-459.

84. Biggio G. et al. - Farmaci del sistema nervoso - Rossi F. et al. - FARMACOLOGIA - Principi di base ed applicazioni terapeutiche - 2005 - Edizioni Minerva Medica - Chapter 3, pp 169-285. 
85. Lundberg J. et al. - Serotonin transporter occupancy with TCAs and SSRIs: a PET study in patients with major depressive disorder. - Int J Neuropsychopharmacol. - 2012 Sep; 15(8): 1167-72.

86. Ruhé HG et al. - Occupancy of serotonin transporters in the amygdala by paroxetine in association with attenuation of the left amygdala activation by negative faces in major depressive disorder - Psychiatry Res. 2014 Feb 28;221(2): 155-61.

87. Lothe A. et al. $-5-\mathrm{HT}\left(1^{\circ}\right)$ receptor binding changes in patients with major depressive disorder before and after antidepressant treatment: a pilot $\left[{ }^{18} \mathrm{~F}\right] \mathrm{MPPF}$ positron emission tomography study - Psychiatry Res. 2012 Jul 30; 203(1): 103-4.

88. Gray NA et al. - Antidepressant treatment reduces serotonin-1 ${ }^{\circ}$ autoreceptor binding in major depressive disorder. - Biol Psychiatry. 2013 Jul 1;74(1):26-31.

89. Hirvonen J. et al. - Effects of antidepressant drug treatment and psychotherapy on striatal and thalamic dopamine D2/3 receptors in major depressive disorder studied with $\left[{ }^{11} \mathrm{C}\right]$ raclopride PET - J Psychopharmacol. 2011 Oct;25(10): 1329-36.

90. Lai Ch et al. - Duloxetine's modest short-term influences in subcortical structures of first episode drug- naïve patients with major depressive disorder and panic disorder - Psychiatry Res. 2011 Nov 30;194(2): 157-62.

91. Kong L. et al. - Frontal-subcortical volumetric deficits in single episode, medication naïve depressed patients and the effects of 8 weeks fluoxetine treatment: a VBM-DARTEL study - PloS One. 2014 Jan 10;9(1):e79055.

92. Marano CM et al. - Structural Imaging in late life depression: association with mood and cognitive responses to antidepressant treatment - Am J Geriatr Psychiatry. 2015 Jan;23(1):4-12.

93. Posner J. et al. - Antidepressants normalize the default mode network in patients with dysthimia - JAMA Psychiatry. 2013 Apr;70(4):373-82.

94. Fu CH. et al. - Multimodal functional and structural neuroimaging investigation of major depressive disorder following treatment with duloxetine - BMC Psychiatry. 2015 Apr 14;15:82.

95. Tao R. et al. - Brain activity in adolescent major depressive disorder before and after fluoxetine treatment Am J Psychiatry. 2012 APr;169(4):381-8.

96. Ruhé HG et al. - Successful pharmacologic treatment of major depressive disorder attenuates amygdala activation to negative facial expressions: a functional magnetic resonance imaging study - J Clin Psychiatry 2012 Apr;73(4):451-9.

97. Arnone D. et al. - Increased amygdala responses to sad but not fearful faces in major depression: relation to mood state and pharmacological treatment - Am J Psychiatry. 2012 Aug;169(8):841-50.

98. Godlewska BR et al. - Short-term SSRI treatment normalises amygdala hyperactivity in depressed patients Psychol Med 2012 Dec;42(12):2609-17.

99. Chen YT et al. - Right and left amygdalae activation in aptients with major depression receiving antidepressant treatment, as revealed by fMRI - Behav Brain Funct. 2014 Oct 8;10(1):36.

100.Wang L. et al. - Short-term effects of escitalopram on regional brain function in first-episode drug-naive patients with major depressive disorder assessed by resting-state functional magnetic resonance imaging Psychol Med. 2014 May;44(7):1417-26.

101.Stoy M. et al - Hyporeactivity of ventral striatum towards incentive stimuli in unmedicated depressed patients normalizes after treatment with escitalopram - J Psychopharmacol. 2012 May;26(5):677-88.

102.Wang L. et al. - The effects of antidepressant treatment on resting-state functional brain networks in patients with major depressive disorder - Hum Brain Mapp. 2015 Feb;36(2):768-78.

103. Yang R. et al. - Hypothalamus-anchored resting brain network changes before and after sertraline treatment in major depression - Biomed Res Int. 2014;2014:915026.

104.Korgaonkar MS et al. - Diffusion tensor imaging predictors of treatment outcomes in major depressive disorder - Br J Psychiatry 2014 Oct;205(4):321-8.

105.Evers Alex S. et al. - Anestetici generali - Brunton L.L. et al. - Goodman and Gilman's The pharmacological Basis of Therapeutics, $11^{\circ}$ edition - Italian Edition - McGrawHill - Chapter 13 - pp 341-367.

106. Fratta W. Et al. - Tossicodipendenza e sostanze d'abuso - Rossi F. et al. - FARMACOLOGIA - Principi di base ed applicazioni terapeutiche - 2005 - Edizioni Minerva Medica - Chapter 16, pp 899-933.

107. Carlson PJ et al. - Neural correlates of rapid antidepressant response to ketamine in treatment-resistant unipolar depression: a preliminary positron emission tomography study. -Biol Psychiatry. 2013 Jun $15 ; 73(12): 1213-21$.

108. Abdallah CG et al. - Hippocampal volume and the rapid antidepressant effect of ketamine - J Psychopharmacol. 2015 May;29(5):591-5.

109. Taylor MJ et al. - Lack of effect of ketamine on cortical glutamate and glutamine in healthy volunteers: a proton magnetic resonance spectroscopy study. - J Psychopharmacol. 2012 May;26(5):733-7.

110. Mylak MS et al. - A pilot in vivo proton magnetic resonance spectroscopy study of amino acid neurotransmitter response to ketamine treatment of major depressive disorder. Mol Psychiatry. 2015 Aug 18.

111. Murrough JW et al. - Regulation of neural responses to emotion perception by ketamine in individuals with treatment-resistant major depressive disorder. - Transl Psychiatry. 2015 Feb 17;5:e509. 
112. Carpiniello B. et al. - Disturbi dell'umore - Biondi Massimo et al. - Manuale di Psichiatria - 2009 - Elsevier - Chapter 5, pp 51-73

113. Saijo et al. - Electroconvulsive therapy decreases dopmine $\mathrm{D}_{2}$ receptor binding in the anterior cingulate in patients with depression: a controlled study using positron emission tomography with radioligand $\left[{ }^{11} \mathrm{C}\right] \mathrm{FLB}$ 457 - J CLin Psychiatry 2010 Jun;71(6):793-9

114.Saijo T et al. - Effect of electroconvulsive therapy on 5-HT1A receptor binding in patients with depression: a PET study with [ ${ }^{11}$ C]WAY 100635 - Int J Neuropsychopharmacol. 2010 Jul;13(6):785-91.

115.Lazemberger $\mathrm{R}$ et al. - Global decrease of serotonin- $1^{\circ}$ receptor binding after electroconvulsive therapy in major depression measured by PET - Mol Psychiatry - 2013 Jan;18(1):93-100

116. Yatham LN et al. - Effect of electroconvulsive therapy on brain 5-HT(2) receptors in major depression - Br J Psychiatry. 2010 Jun;196(6):474-9.

117. Ota $\mathrm{M}$ et al. - Effect of electroconvulsive therapy on gray matter volume in major depressive disorder - $\mathrm{J}$ Affect Disord. 2015 Nov 1;186:186-91.

118. Bouckaert $\mathrm{F}$ et al. - Grey matter volume increase following electroconvulsive therapy in patients with late life depression: a longitudinal MRI study - J Psychiarty Neurosci. 2015 Sep 22;40(5):140322.

119.Tendolkar I et al. - Electroconvulsive therapy increases hippocampal and amygdala volume in therapy refractory depression: a longitudinal pilot study - Psychiatry Res. 2013 Dec 30;214(3):197-203.

120. Ten Doesschate F et al. - Pre-treatment amygdala volume predicts electroconvulsive therapy response - Fornt Psychiatry. 2014 Nov 26;5:169.

121.Nordanskog $\mathrm{P}$ et al. - Hippocampal volume in relation to clinical and cognitive outcome after electroconvulsive therapy in depression - Acta psychiatr Scand. 2014 Apr;129(4):303-11.

122. Abbott CC et al. - Hippocampal strucutral and functional changes associated with electroconvulsive therapy response - Transl Psychiatry 2014 Nov 18;4:e483.

123.Cano $\mathrm{M}$ et al. - Modulation of Limbic and Prefrontal Connectivity by Electroconvulsive Therapy in Treatment-resistant Depression: A Preliminary Study - Brain Stimul. 2015 Sep 1. Pii: S1935-861X(15)011171.

124.Perrin JS et al. - Electroconvulsive therapy reduces frontal cortical connectivity in severe depressive disorder. - Proc Nati Acad Sci U.S.A. 2012 Apr 3;109(14):5464-8.

125.Liu Y et al. - Antidepressant Effects of Electroconvulsive Therapy Correlate With Subgenual Anterior Cingulate Activity and Connectivity in Depression - Medicine (Baltimore). 2015 Nov;94(45):e2033.

126. Beall EB et al. - Effects of lectroconvulsie therapy on brain functional activation and connectivity in depression - J ECT 2012 Dec:28(4):234-41.

127. Baeken $\mathrm{C}$ et al. - HF-rTMS treatment in medication resistant melancholic depression: results from 18FDGPET brain imaging. - CNS Spectr. 2009 Aug;14(8):439-48.

128.Furtado CP et al. - Cognitive and volumetric predictors of response to repetitive transcranial magnetic stimulation (rTMS) - a prospective follow-up study. - Psychiatry Res. 2012 Apr 30;202(1):12-9.

129.Hernández-Ribas R et al. - Identifying brain imaging correlates of clinical response to repetitive transcranial magnetic stimulation (rTMS) in major depression. - Brain Stimul. 2013 Jan;6(1):54-61.

130. Mackin RS et al. - Patterns of reduced cortical thickness in late-life depression and relationship to psychotherapeutic response - Am J Geriatr Psychiatry. 2013 Aug;21(8):794-802.

131. Garrett AS et al. - Changes in brain activation following psychotherapy for youth with mood dysregulation at familial risk for bipolar disorder - Prog Neuropsychopharmacol Biol Psychiatry. 2015 Jan 2;56:215-20.

132. Buchheim A. et al. - Changes in prefrontal-limbic function in major depression after 15 months of long-term psychotherapy - PloS One. 2012;7(3)e33745.

133.Ives-Deiperi VL et al. - The effects of mindfulness-based cognitive therapy in patients with bipolar disorder: a controlled functional MRI investigation. - J Affect Disord. 2013 Sep 25;150(3):1152-7.

134. Straub J. et al - Neural correlates of successful psychotherapy of depression in adolescents - J Affect Disord. 2015 Sep 1;183:239-46.

135.Klein JP et al. - Effect of specific psychotherapy for chronic depression on neural responses to emotional faces - J Affect Disord. 2014 Sep;166:93-7.

136. Huang $\mathrm{X}$ et al. - Early brain changes associated with psychotherapy in major depressive disorder revealed by resting-state fMRI: evidence for the top-down regulation theory - Int J Psychophysiol. 2014 Dec;94(3):437-44

137. Yuan $\mathrm{H}$ et al. - Resting-state functional connectivity modulation and sustained changes after real-time functional magnetic resonance imaging neurofeedback training in depression - Brain Connect. 2014 Nov;4(9):690-701 